\title{
Single nucleotide polymorphisms at the TRAF1/C5 locus are associated with rheumatoid arthritis in a Han Chinese population
}

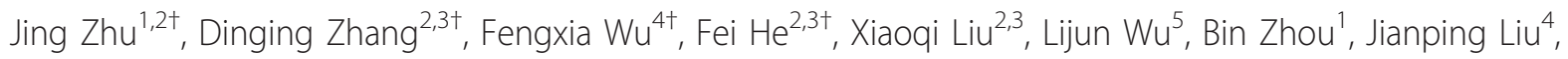
Fang Lu ${ }^{2,3}$, Jian Liu', Ruijun Luo ${ }^{2}$, Wubin Long ${ }^{1}$, Minghui Yang ${ }^{4}$, Shi Ma ${ }^{2,3}$, Xiaodan Wu', Yi Shi ${ }^{2,3}$, Tong Wu', Ying Lin ${ }^{2,3}$, Jiyun Yang ${ }^{2,3}$, Guohua Yuan ${ }^{4}$ and Zhenglin Yang ${ }^{2,3^{*}}$

\begin{abstract}
Background: Genetic variants in TRAF1C5 and PTPN22 genes have been shown to be significantly associated with arthritis rheumatoid in Caucasian populations. This study investigated the association between single nucleotide polymorphisms (SNPS) in TRAF1/C5 and PTPN22 genes and rheumatoid arthritis (RA) in a Han Chinese population. We genotyped SNPs rs3761847 and rs7021206 at the TRAF1/C5 locus and rs2476601 SNP in the PTPN22 gene in a Han Chinese cohort composed of 576 patients with RA and 689 controls. The concentrations of anti-cyclic citrullinated peptide antibodies (CCP) and rheumatoid factor (RF) were determined for all affected patients. The difference between the cases and the controls was compared using $X^{2}$ analysis.

Results: Significant differences in SNPs rs3761847 and rs7021206 at TRAF1/C5 were observed between the case and control groups in this cohort; the allelic p-value was 0.0018 with an odds ratio of 1.28 for rs3761847 and 0.005 with an odds ratio of 1.27 for rs7021206. This significant association between rs3761847 and RA was independent of the concentrations of anti-CCP and RF. No polymorphism of rs 2476601 was observed in this cohort.

Conclusions: We first demonstrated that genetic variants at the TRAF1/C5 locus are significantly associated with RA in Han Chinese, suggesting that TRAF1/C5 may play a role in the development of RA in this population, which expands the pathogenesis role of TRAF1/C5 in a different ethnicity.
\end{abstract}

Keywords: rheumatoid arthritis genetics, TRAF1/C5, association study, Chinese

\section{Background}

Rheumatoid arthritis (RA) is a chronic inflammatory disease that affects about $1 \%$ of the adult Caucasian population and $0.37 \%$ of the Chinese population. Women are affected three times more often than men [1-3]. This disease may affect many tissues and organs; it particularly destroys synovial joints involving autoimmune features [2]. The disease can cause severe disability and even early mortality.

Although the full etiology of RA remains unclear, it is considered a complex disease caused by the interaction

\footnotetext{
* Correspondence: zliny@yahoo.com

+ Contributed equally

${ }^{2}$ Center for Human Molecular Biology \& Genetics, Sichuan Academy of Medical Sciences \& Sichuan Provincial People's Hospital, Chengdu, Sichuan, China

Full list of author information is available at the end of the article
}

of genetic variants, the environment, infectious and hormonal factors [4]. The genetic variants may contribute $50-60 \%$ of the etiology of RA [5]. At least 31 RA risk loci have been confirmed associated with RA in different populations [6-14]. At TRAF1/C5 locus, both TRAF1 and $\mathrm{C} 5$ are possible RA-causing genes due to their biological functions. TNF is a critical cytokine in the pathogenesis of RA [15]. TRAF1 and TRAF2 form a heterodimeric complex, which is required for TNFalpha-mediated activation of MAPK8/JNK and NF-kap$\mathrm{paB}$. The protein complex formed by this protein and TRAF2 also interacts with inhibitor-of-apoptosis proteins (IAPs), and thus mediates the anti-apoptotic signals from TNF receptors. TNF antagonists are an effective treatment for rheumatoid arthritis [15-17]. On the other hand, the clinical and biological data for $\mathrm{C} 5$
C Biomed Central

() 2011 Zhu et al; licensee BioMed Central Ltd. This is an Open Access article distributed under the terms of the Creative Commons Attribution License (http://creativecommons.org/licenses/by/2.0), which permits unrestricted use, distribution, and reproduction in any medium, provided the original work is properly cited. 
are equally compelling [15]. The complement pathway has been implicated in the pathogenesis of RA for a long time $[18,19]$. Complement activation leading to significant depletion of complement components has been shown in synovial fluid of patients with RA [15]. rs7021206 is located at TRAF1 intron 3 and rs3761847 is located at the upstream of TRAF1 and the down stream of $\mathrm{C} 5$. These variants or other causative variants at this locus may affect the function or expression levels of TRAF1 and/or C5 to lead to RA.

In this study, we investigated the association between rs7021206 and rs3761847 at the TRAF1/C5 locus and rs2476601 in the PTPN22 gene and RA in a Han Chinese population.

\section{Methods \\ Patients}

The Institutional Review Boards of the Sichuan Academy of Medical Sciences \& Provincial People's Hospital, North Sichuan Medical College, China, approved this study. All subjects provided informed consent before participating in the study. RA patients and normal matched controls, including individuals with a normal joint examination, were recruited at the rheumatology and Immunology clinics at Sichuan Provincial People's Hospital and North Sichuan Medical College, China. All participants went through a standard examination protocol as noted in the previous description: (http://medcalc3000.com/RheumatoidArthritis.htm, Rheumatoid Arthritis Criteria 1987 revision, American Rheumatism Association), including morning stiffness, arthritis of three or more joints, including hand joints, symmetric arthritis, rheumatoid nodules, serum rheumatoid factor, and radiographic changes. In total, 576 patients with RA and 689 normal age-matched controls were recruited. All patients enrolled in this study at least two years after clinical diagnosis of RA and the patients had five years of mean disease duration. In the normal controls, no sign of joint or other autoimmune disease was detected. Clinical information about the cases and controls is listed in Table 1.

\section{$\mathrm{CCP}$ and RF measurement}

Sera were obtained and tested for concentrations of RF and anti-CCP in all patients. The concentrations of antiCCP antibodies were detected with an Anti-CCP-ELISA kit (EUROIMMUN Medizinische Labordiagnostika AG,
Lübeck, Germany) on a Bio Rad Bench Mark machine (Rio Rad, Hercules, CA, USA). RF was determined with an endpoint nephelometry kit (Siemens Healthcare Diagnostics Products GmbH, Marburg, Germany) on a DADE BEHRING BNII machine (GMI, Minnesota, USA) according to the manufacturer's manual instructions. Results were corroborated by validation at the clinical laboratory of Sichuan Provincial People's Hospital, Sichuan, China. Cases with anti-CCP levels higher than 20 units/ml were considered positive for anti-CCP antibodies; cases with RF levels higher than 5 units $/ \mathrm{ml}$ were considered positive for RF.

\section{Genotyping}

Based on previous genome-wide association studies (GWAS) and replication studies in Asians [6-13,20], we selected rs7021206 and rs3761847 at the TRAF1/C5 locus and rs2476601 in the PTPN22 gene to genotype in the Han Chinese population. Venous blood from each subject was withdrawn and collected in an EDTA tube. Genomic DNA was extracted from the blood by serial phenol/chloroform extraction and ethanol precipitation. SNP genotyping was performed with the dye terminator-based SNaPshot method (Applied Biosystems, CA, USA). The SNP reported in this manuscript has a genotyping success rate of $97 \%$ and accuracy as judged by random re-genotyping of $10 \%$ of the samples in the cohort. For rs3761847, the PCR forward primer 5'CCTACCTGTTCCCTCCTTCC-3', PCR reverse primer 5'-GGGATGATGATGGCAATACC-3', and SNaPshot primer 5'- AGGTAGAGAGGGTGGTATTGAGGC-3' were used in genotyping. For rs7021206, the PCR forward primer 5'-GAGAAGCAGATGGGAGAGTG-3', PCR reverse primer 5'-TGCTTGGCTAGCAGCAATTC-3', and SNaPshot primer 5'-AAGGAGAAGGAGGTGTGGACACTCGCCTGCCTCTGGTCCA-3' were used in genotyping. For rs2476601, the PCR forward primer 5'- GGCCTCAATGAACTCCTCAA-3', PCR reverse primer 5'- GGATAGCAACTGCTCCAAGG-3'and SNaPshot primer 5'-CCTCAACCACAATAAATGATTCAGGTGTCC-3' were used in genotyping.

\section{Statistical analysis}

The Hardy-Weinberg equilibrium (HWE) for the SNP was tested with the $\chi^{2}$ test. All analyses were adjusted for matching factors of age and sex factors. Allele and

Table 1 Characteristics of RA cases and controls matched for ages and ethnicity

\begin{tabular}{ccccccc}
\hline Subject & Total number & anti-CCP positive & RF positive & Male & Female & Average age \\
\hline Cases & 576 & 363 & 496 & 266 & 310 & $64 \pm 6.6$ \\
Controls & 689 & 0 & 0 & 307 & 382 & $65 \pm 7.8$ \\
\hline
\end{tabular}

\pm represents standard deviation. 
genotype frequencies between cases and controls were compared with the $\chi^{2}$ analysis. Statistical significance was defined as $\mathrm{p}<0.05$. All statistical analyses were performed using the software SPSS version 10.0.

\section{Results}

Among the 576 patients with RA, 363 of them were anti-CCP positive and 496 of them were RF positive (Table 1). Linkage disequlibrium between rs3761847 and rs7021206 was $0.23\left(r^{2}\right)$ in the Chinese cohort studied. Allele distributions both of rs3761847 and rs7021206 were within the Hardy-Weinberg equilibrium in both the case and control groups ( $p>0.05$, Table 2). We found that both rs3761847 and rs7021206 were significantly associated with RA in the cohort studied (Allelic $\mathrm{p}=0.0018$, adjusted $\mathrm{p}=0.054$ for $\mathrm{rs} 3761847$; allelic $\mathrm{p}=$ 0.005 , adjusted $\mathrm{p}=0.015$ for rs7021206, Table 2). For rs3761847, the risk allele frequency was 0.58 in the patients and 0.52 in the controls. The risk allele of this SNP conferred a 1.28-fold (95\% CI: 1.10 1.50) increased likelihood of RA (Table 2). For rs7021206, the risk allele frequency was 0.34 in the patients and 0.29 in the controls. The risk allele of this SNP conferred a 1.27-fold (95\% CI: 1.07 1.50) increased likelihood of RA (Table 2). rs3761847 showed significant association with RA in both the anti-CCP positive and anti-CCP negative groups. The $\mathrm{p}$ value is 0.0015 in the anti-CCP positive group with an odds ratio (OR) of 1.33 (95\% CI: 1.111.60; Table 3); the $\mathrm{p}$ value is 0.043 in the anti-CCP negative group with an OR of 1.25 (95\% CI: 1.0-1.56; Table 3). rs3761847 also showed a significant association with RA in both the RF positive and negative groups. The $\mathrm{p}$ value was 0.0015 in the RF positive group with an OR of 1.30 (95\% CI: 1.10-1.53; Table 3); the p value was 0.03 in the RF negative group with an OR of 1.44 (95\% CI: 1.03-2.01; Table 3). rs7021206 also showed significant association with RA in both the anti-CCP positive and RF positive groups, $\left(\mathrm{p}=2.5 \times 10^{-4}\right.$ and 1.4 $\times 10^{-4}$, respectively, table 3 ), but not in anti-CCP or RF negative groups ( $\mathrm{p}=0.065,0.29$, respectively, table 3 ).

No polymorphism was observed for rs2476601 in the cohort.

\section{Discussion}

Although at least 31 susceptibility loci/genes responsible for RA have been identified [14]; including HLA-DRB1 $([7,13]$, protein tyrosine phosphatase 22 (PTPN22) gene [13,21], tumor necrosis factor, alpha-induced protein 3 (TNFAIP3) locus on chromosome 6q [8,9,13], TRAF1/ C5 [10,11], STAT4 [12], interferon regulatory factor 5 isoform a (IRF5) [22], peptidylarginine deiminase (PADI4) [23], CD244 natural killer cell receptor 2B4 (CD244) [24], CD40 antigen isoform 2 precursor (CD40) [25], small inducible cytokine A21 precursor (CCL21) [25], and solute carrier family 22 member 4 (SLC22A4) [26] and others [14], only 5 loci/genes (HLA, PTPN22, TRAF1/C5, TNFAIP3, and STAT4) have been successfully replicated in different ethnicities, whereas others have been observed less systematically or further replication studies in different ethnicities are needed. Among the five successfully replicated loci, $H L A$ showed a significant association with RA in all different ethnicities. The association of STAT4 with RA has also been successfully replicated in Asians, including Japanese, Koreans and Chinese [27-29]; and the association of TRAF1/C5 with RA has also been replicated in Japanese and Korean populations [20,30].

Although rs2476601 (R620W) in PTPN22 has been constantly associated with RA in populations of European descent, including US [31], UK [32], Finnish [33], Swedish [34], German [35], Dutch [36], Spanish [37], and Canadian [38] populations, this SNP did not show a significant association with RA in the Japanese population [39] studied because there is a very low minor allele

Table 2 Association between rs3761847 and rs7021206 in TRAF1/C5 and RA in a Han Chinese cohort

\begin{tabular}{|c|c|c|c|c|c|c|c|}
\hline SNP & Phenotype & HWE & Genotype count (frequency) & Allele frequency & Allelic $p$ & Adjusted $\mathrm{p}$ & OR $(95 \% \mathrm{Cl})$ \\
\hline & Case (576) & 0.93 & AA: 192 (0.33) & A:0.58 & 0.0018 & 0.0054 & $1.28(1.10-1.50)$ \\
\hline & & & AG: $282(0.49)$ & G:0.42 & & & \\
\hline \multirow[t]{6}{*}{ rs3761847 } & & & GG: 102 (0.18) & & & & \\
\hline & Control (689) & 0.06 & AA: $171(0.25)$ & $A: 0.52$ & & & \\
\hline & & & AG: 369 ( 0.54) & G:0.48 & & & \\
\hline & & & GG:149 ( 0.21) & & & & \\
\hline & Case (576) & 0.07 & AA: $239(0.41)$ & A:0.66 & 0.005 & 0.015 & $1.27(1.07-1.50)$ \\
\hline & & & AG: $279(0.48)$ & G:0.34 & & & \\
\hline \multirow[t]{4}{*}{ rs7021206 } & & & GG: 58 (0.11) & & & & \\
\hline & Control (689) & 0.54 & AA: $343(0.50)$ & A:0.71 & & & \\
\hline & & & AG: 291 (0.42) & G:0.29 & & & \\
\hline & & & GG: 55 ( 0.08 ) & & & & \\
\hline
\end{tabular}

SNP: single nucleotide polymorphism, HWE: Hardy-Weinberg equilibrium, OR: odds ratio, Cl: confidence interval. 
Table 3 Association of rs3761847 and rs7021206 in TRAF1/C5 and subgroup RA in a Han Chinese cohort

\begin{tabular}{|c|c|c|c|c|c|c|c|c|}
\hline \multirow[t]{2}{*}{ SNP } & \multirow[t]{2}{*}{ Group } & \multirow[t]{2}{*}{ HWE } & \multicolumn{2}{|c|}{ Genotype count and frequency } & \multicolumn{2}{|c|}{ Allele frequency } & \multirow[t]{2}{*}{ Allelic $p$} & \multirow[t]{2}{*}{ OR $(95 \% \mathrm{Cl})$} \\
\hline & & & Case (frequency) & Control (frequency) & Case & Control & & \\
\hline & anti-CCP positive & Case: 0.76 & $A A: 127(0.35)$ & $A A: 171(0.25)$ & A: 0.59 & A: 0.52 & 0.0015 & $1.33(1.11-1.60)$ \\
\hline & & Control: 0.058 & AG:173 (0.48) & $A G: 369(0.54)$ & G: 0.41 & G: 0.48 & & \\
\hline & & & GG:63 (0.17) & $G G: 149(0.21)$ & & & & \\
\hline & anti-CCP negative & Case: 0.81 & $A A: 69(0.32)$ & $A A: 171(0.25)$ & A: 0.57 & A: 0.52 & 0.040 & $1.25(1.01-1.56)$ \\
\hline & & Control: 0.058 & AG:106 (0.50) & $A G: 369(0.54)$ & G: 0.43 & $G: 0.48$ & & \\
\hline \multirow[t]{12}{*}{ rs3761847 } & & & GG:38 (0.18) & $G G: 149(0.21)$ & & & & \\
\hline & RF positive & Case: 0.81 & $A A: 170(0.34)$ & $A A: 171(0.25)$ & A: 0.58 & A: 0.52 & 0.0015 & $1.30(1.10-1.53)$ \\
\hline & & Control: 0.058 & AG:237 (0.48) & $A G: 369(0.54)$ & $G: 0.42$ & G: 0.48 & & \\
\hline & & & GG:89 (0.18) & $G G: 149(0.21)$ & & & & \\
\hline & RF negative & Case: 0.26 & $A A: 27(0.34)$ & $A A: 171(0.25)$ & A: 0.61 & A: 0.52 & 0.030 & $1.44(1.03-2.01)$ \\
\hline & & Control: 0.058 & AG:43 (0.54) & $A G: 369(0.54)$ & G: 0.39 & G: 0.48 & & \\
\hline & & & GG:10 (0.16) & GG:149 (0.21) & & & & \\
\hline & anti-CCP positive & Case: 0.22 & $A A: 139(0.38)$ & $A A: 343(0.50)$ & G: 0.37 & G: 0.29 & 0.00025 & $1.42(1.18-1.72)$ \\
\hline & & Control: 0.54 & $A G: 180(0.50)$ & AG:291 (0.42) & A: 0.63 & $A: 0.71$ & & \\
\hline & & & GG:44 (0.12) & GG:55 ( 0.08$)$ & & & & \\
\hline & anti-CCP negative & Case: 0.18 & $A A: 89(0.42)$ & $A A: 343(0.50)$ & G: 34 & G: 0.29 & 0.065 & \\
\hline & & Control: 0.54 & AG:104 (0.49) & $A G: 291(0.42)$ & A: 0.66 & A: 0.71 & & \\
\hline \multirow[t]{7}{*}{ rs7021206 } & & & GG:20 (0.09) & GG:55 ( 0.08$)$ & & & & \\
\hline & RF positive & Case: 0.17 & AA:193 (0.39) & $A A: 343(0.50)$ & G: 0.36 & G: 0.29 & 0.00014 & $1.40(1.18-1.67)$ \\
\hline & & Control: 0.54 & $A G: 244(0.49)$ & $A G: 291(0.42)$ & A: 0.64 & $A: 0.71$ & & \\
\hline & & & GG:59 (0.12) & GG:55 ( 0.08$)$ & & & & \\
\hline & RF negative & Case: 0.26 & $A A: 38(0.48)$ & $A A: 343(0.50)$ & G: 0.33 & G: 0.29 & 0.29 & \\
\hline & & Control: 0.54 & AG:31 (0.39) & AG:291 (0.42) & A: 0.67 & $A: 0.71$ & & \\
\hline & & & $G G: 11(0.13)$ & GG:55 ( 0.08$)$ & & & & \\
\hline
\end{tabular}

SNP: single nucleotide polymorphism, HWE: Hardy-Weinberg equilibrium, OR: odds ratio, Cl: confidence interval.

frequency in this population. In addition, although tag SNPs covering the PTPN22 linkage disequilibrium block were polymorphic in the Korean population, Lee et al did not reveal any disease association, and the resequencing did not identify any new common coding region variants in this population [40]. Consistent with results in Japanese and Korean populations, we did not find even a single minor allele of rs2476601 in the studied Chinese cohort, suggesting that rs2476601 in PTPN22 is not associated with RA in Asians.

On the other hand, genetic variants in PADI4 have shown significant association with RA in Asian populations $[24,41,42]$ but not in Caucasian populations [34,43]. Therefore, we believe the genetics of RA shows obvious ethnic differences.

rs3761847 at the TRAF1/C5 locus has been significantly associated with RA in an initial genome-wide association study (GWAS) in a North American and Swedish cohort, composed of 1493 cases and 1831 controls [11]. This finding was replicated in the second cohort composed of 485 cases and 1282 controls in a North American population [11]. In addition, using a candidate gene approach, Kurreeman et al identified that rs10818488 in TRAF1/C5 is significantly associated with RA in patients of Dutch origin [10]. This finding was confirmed in a case-control study in the population of the island of Crete, Greece [44] and a meta-analysis [45]. Kurreeman et al also confirmed the TRAF1/C5 locus as a susceptibility locus for rheumatoid arthritis in a European family-based replication study using rs10818488 at this region [46]. However, this association could not be confirmed in a Swedish cohort composed of 568 cases and 516 controls. Chang et al [15], carried out a multi-tiered, case-controlled association study, genotyping 25,966 putative functional SNPs in an initial North America cohort, composed of 475 cases and 475 controls and two replication cohorts (661 cases/1322 controls from North America and 596 cases/705 controls from the Netherlands). The authors identified an SNP, rs1953126 at the TRAF1/C5 locus that was significantly associated with $\mathrm{RA}(\mathrm{OR}=1.28$, trend $\mathrm{p}=1.45 \times$ $\left.10^{-6}\right)$. Through a comprehensive fine-scale-mapping study, they found a variety of analyses identified SNPs in a $70 \mathrm{~kb}$ region extending from the third intron of PHF19 across TRAF1 into the TRAF1-C5 intergenic region, excluding the $C 5$ coding region. This study 
suggested that TRAF1 is the RA gene at the TRAF1-C5 locus, but further studies are needed to confirm this conclusion.

In recent studies, rs3761847 has been shown to be significantly associated with RA in Japanese [30]. Our results further confirmed that rs3761847 at the TRAF1/ C5 was associated with RA in Han Chinese Asians, and this association might not depend on the concentrations of anti-CCP antibodies or RF. Thus, TRAF1/C5 is another locus playing a role in RA development in different ethnicities. We observed that rs3761847 A increased the risk for RA in the present study, which is consistent with the results observed in a Japanese population [30], however, this is the opposite of the original studies in Caucasian populations, in which rs3761847 A decreased the risk of RA. In addition, rs3761847 did not show a significant association with RA in the Korean population $[20,30]$. On the other hand, we found that rs7021206 at intron 3 of TRAF1 was showed significant association with RA in the Han Chinese in this study, which is consistent with previous studies in Korean and Caucasian populations [20]. This indicated that rs7021206, but not rs376184, may be the true causative allele at the TRAF1-C5 locus [20,47]; However, the true causative allele in this region needs to be further investigated.

The significant association between rs3761847 and RA was observed in both anti-CCP and RF positive groups and negative groups, suggesting that this significant association between TRAF1-C1 and RA was independent of the concentrations of anti-CCP and RF [20]. No significant association was observed between rs7021206 and RA in anti-CCP and RF negative groups; this may be because of small sample size in these groups in the current studies.

Other studies indicate genetic variants at the TRAF1/ C5 locus are associated with autoimmune diseases affecting multiple organs, such as systemic lupus erythematous (SLE) $[48,49]$, and juvenile idiopathic arthritis [50-52]. Thus, the functional study of TRAF1/C5 with autoimmune disease may provide an insight into the pathogenesis and treatment of these diseases as well as RA.

\section{Conclusions}

We first demonstrated that genetic variants rs3761847 and rs7021206 in TRAF1/C5 locus are significantly associated with RA in the Han Chinese, suggesting that TRAF1/C5 may play a role in the development RA in this population, which expands the pathogenesis role of TRAF1/C5 in a different ethnicity. Unlike in Caucasian populations, rs2476601 in the PTPN22 gene did not show polymorphism in the Han Chinese population being studied, suggesting that either other genetic variants in this gene are associated with RA in the Han
Chinese population or PTPN22 has nothing to do with RA the population.

\section{Acknowledgements}

We thank the participating RA patients and their families. The authors acknowledge the following grant support: Department of Science and Technology of Sichuan Province grant 08ZC0479 ( to Z. Yang) and Department of Public Health of Sichuan Province grants 080326 (to J. Zhu) and 070062 (to Z. Yang).

\section{Author details}

1Department of Rheumatology and Immunology, Sichuan Academy of Medical Sciences \& Sichuan Provincial People's Hospital, Chengdu, Sichuan, China. ${ }^{2}$ Center for Human Molecular Biology \& Genetics, Sichuan Academy of Medical Sciences \& Sichuan Provincial People's Hospital, Chengdu, Sichuan, China. ${ }^{3}$ Institute of Laboratory Medicine, Sichuan Academy of Medical Sciences \& Sichuan Provincial People's Hospital, Chengdu, Sichuan, China. ${ }^{4}$ Institute of Rheumatology and Immunology, Affiliated Hospital, North Sichuan Medical College, Nanchong, Sichuan, China. ${ }^{5}$ Department of Rheumatology, People's Hospital of Xin Jiang Uygur Autonomous Region, Urumchi, Xin Jiang, China.

\section{Authors' contributions}

Designed the study: ZY and JZ; collected samples and experiments: JZ, DZ, $F W, L W, B Z, J L, F L, J L, R L, W L, M Y, S M, X W, Y S, T W, Y L, J Y, G Y$ and $Z Y$; performed the data analysis: $X L$ and $Z Y$; writing the manuscript: $Z Y$. All authors read and approved the final manuscript.

\section{Competing interests}

The authors declare that they have no competing interests.

Received: 10 January 2010 Accepted: 14 April 2011

Published: 14 April 2011

\section{References}

1. Silman AJ, Pearson JE: Epidemiology and genetics of rheumatoid arthritis. Arthritis Res 2002, 4(Suppl 3):S265-72.

2. Majithia $V$, Geraci SA: Rheumatoid arthritis: Diagnosis and management. Am J Med 2007, 120:936-9.

3. Xiang YD, SM: Rheumatology international. 2009, 29, (no.5).

4. Firestein GS: Evolving concepts of rheumatoid arthritis. Nature 2003, 423:356-61.

5. MacGregor AJ, Snieder H, Rigby AS, et al: Characterizing the quantitative genetic contribution to rheumatoid arthritis using data from twins. Arthritis Rheum 2000, 43:30-7.

6. Bowes J, Barton A: Recent advances in the genetics of ra susceptibility. Rheumatology (Oxford) 2008, 47:399-402.

7. Gregerson PK, Silver J, Winchester : Arthritis Rheum. 1987, 30:1205-13.

8. Thomson W, Barton A, Ke X, et al: Rheumatoid arthritis association at 6q23. Nat Genet 2007, 39:1431-3.

9. Plenge RM, Cotsapas C, Davies L, et al: Two independent alleles at $6 q 23$ associated with risk of rheumatoid arthritis. Nat Genet 2007, 39:1477-82.

10. Kurreeman FA, Padyukov L, Marques RB, et al: A candidate gene approach identifies the traf1/c5 region as a risk factor for rheumatoid arthritis. PLoS Med 2007, 4:e278.

11. Plenge RM, Seielstad M, Padyukov L, et al: Traf1-c5 as a risk locus for rheumatoid arthritis-a genomewide study. N Engl J Med 2007, 357:1199-209.

12. Remmers EF, Plenge RM, Lee AT, et al: Stat4 and the risk of rheumatoid arthritis and systemic lupus erythematosus. N Engl J Med 2007, 357:977-86.

13. Genome-wide association study of 14,000 cases of seven common diseases and 3,000 shared controls. Nature 2007, 447:661-78.

14. Stahl EA, Raychaudhuri S, Remmers EF, et al: Genome-wide association study meta-analysis identifies seven new rheumatoid arthritis risk loci. Nat Genet 42:508-14.

15. Chang M, Rowland CM, Garcia VE, et al: A large-scale rheumatoid arthritis genetic study identifies association at chromosome 9q33.2. PLoS Genet 2008, 4:e1000107. 
16. Elliott MJ, Maini RN, Feldmann M, et al: Randomised double-blind comparison of chimeric monoclonal antibody to tumour necrosis factor alpha (ca2) versus placebo in rheumatoid arthritis. Lancet 1994, 344:1105-10.

17. Weinblatt ME, Kremer JM, Bankhurst AD, et al: A trial of etanercept, a recombinant tumor necrosis factor receptor:Fc fusion protein, in patients with rheumatoid arthritis receiving methotrexate. N Engl J Med 1999, 340:253-9.

18. Cooke TD, Hurd ER, Jasin HE, Bienenstock J, Ziff M: Identification of immunoglobulins and complement in rheumatoid articular collagenous tissues. Arthritis Rheum 1975, 18:541-51.

19. Plenge R, Rioux JD: Identifying susceptibility genes for immunological disorders: Patterns, power, and proof. Immunol Rev 2006, 210:40-51.

20. Han TU, Bang SY, Kang C, Bae SC: Traf1 polymorphisms associated with rheumatoid arthritis susceptibility in asians and in caucasians. Arthritis Rheum 2009, 60:2577-84.

21. Hinks A, Worthington J, Thomson W: The association of ptpn22 with rheumatoid arthritis and juvenile idiopathic arthritis. Rheumatology (Oxford) 2006, 45:365-8

22. Sigurdsson S, Padyukov L, Kurreeman FA, et al: Association of a haplotype in the promoter region of the interferon regulatory factor 5 gene with rheumatoid arthritis. Arthritis Rheum 2007, 56:2202-10.

23. Suzuki A, Yamada R, Chang $X$, et al: Functional haplotypes of padi4, encoding citrullinating enzyme peptidylarginine deiminase 4 , are associated with rheumatoid arthritis. Nat Genet 2003, 34:395-402.

24. Suzuki A, Yamada R, Kochi $Y$, et al: Functional snps in cd244 increase the risk of rheumatoid arthritis in a japanese population. Nat Genet 2008, 40:1224-9.

25. Raychaudhuri S, Remmers EF, Lee AT, et al: Common variants at $\mathrm{cd} 40$ and other loci confer risk of rheumatoid arthritis. Nat Genet 2008, 40:1216-23.

26. Tokuhiro $S$, Yamada $R$, Chang $X$, et al: An intronic snp in a runx 1 binding site of slc22a4, encoding an organic cation transporter, is associated with rheumatoid arthritis. Nat Genet 2003, 35:341-8.

27. Kobayashi S, Ikari K, Kaneko H, et al: Association of stat4 with susceptibility to rheumatoid arthritis and systemic lupus erythematosus in the japanese population. Arthritis Rheum 2008, 58:1940-6.

28. Li H, Zou Q, Xie Z, et al: A haplotype in stat4 gene associated with rheumatoid arthritis in caucasians is not associated in the han chinese population, but with the presence of rheumatoid factor. Rheumatology (Oxford) 2009, 48:1363-8.

29. Lee HS, Remmers EF, Le JM, Kastner DL, Bae SC, Gregersen PK: Association of stat4 with rheumatoid arthritis in the korean population. Mol Med 2007, 13:455-60.

30. Nishimoto K, Kochi Y, Ikari K, et al: Association study of traf1-c5 polymorphisms with susceptibility to rheumatoid arthritis and systemic lupus erythematosus in japanese. Ann Rheum Dis 2009.

31. Begovich $A B$, Carlton $V E$, Honigberg $L A$, et al: $A$ missense single-nucleotide polymorphism in a gene encoding a protein tyrosine phosphatase (ptpn22) is associated with rheumatoid arthritis. Am J Hum Genet 2004, 75:330-7.

32. Hinks A, Barton A, John S, et al: Association between the ptpn22 gene and rheumatoid arthritis and juvenile idiopathic arthritis in a uk population: Further support that ptpn22 is an autoimmunity gene. Arthritis Rheum 2005, 52:1694-9.

33. Seldin MF, Shigeta R, Laiho $K$, et al: Finnish case-control and family studies support ptpn22 r620 w polymorphism as a risk factor in rheumatoid arthritis, but suggest only minimal or no effect in juvenile idiopathic arthritis. Genes Immun 2005, 6:720-2.

34. Plenge RM, Padyukov L, Remmers EF, et al: Replication of putative candidate-gene associations with rheumatoid arthritis in $>4,000$ samples from north america and sweden: Association of susceptibility with ptpn22, ctla4, and padi4. Am J Hum Genet 2005, 77:1044-60.

35. Pierer M, Kaltenhauser S, Arnold S, et al: Association of ptpn22 1858 single-nucleotide polymorphism with rheumatoid arthritis in a german cohort: Higher frequency of the risk allele in male compared to female patients. Arthritis Res Ther 2006, 8:R75.

36. Zhernakova A, Eerligh P, Wijmenga C, Barrera P, Roep BO, Koeleman BP: Differential association of the ptpn22 coding variant with autoimmune diseases in a dutch population. Genes Immun 2005, 6:459-61.

37. Orozco G, Sanchez E, Gonzalez-Gay MA, et al: Association of a functional single-nucleotide polymorphism of ptpn22, encoding lymphoid protein phosphatase, with rheumatoid arthritis and systemic lupus erythematosus. Arthritis Rheum 2005, 52:219-24.

38. van Oene M, Wintle RF, Liu X, et al: Association of the lymphoid tyrosine phosphatase r620w variant with rheumatoid arthritis, but not crohn's disease, in canadian populations. Arthritis Rheum 2005, 52:1993-8.

39. Ikari $\mathrm{K}$, Momohara $\mathrm{S}$, Inoue $\mathrm{E}$, et al: Haplotype analysis revealed no association between the ptpn22 gene and ra in a japanese population. Rheumatology (Oxford) 2006, 45:1345-8.

40. Lee HS, Korman BD, Le JM, et al: Genetic risk factors for rheumatoid arthritis differ in caucasian and korean populations. Arthritis Rheum 2009, 60:364-71.

41. Ikari K, Kuwahara M, Nakamura T, et al: Association between padi4 and rheumatoid arthritis: A replication study. Arthritis Rheum 2005, 52:3054-7.

42. Kang CP, Lee HS, Ju H, Cho H, Kang C, Bae SC: A functional haplotype of the padi4 gene associated with increased rheumatoid arthritis susceptibility in koreans. Arthritis Rheum 2006, 54:90-6.

43. Lee YH, Rho YH, Choi SJ, Ji JD, Song GG: Padi4 polymorphisms and rheumatoid arthritis susceptibility: A meta-analysis. Rheumatol Int 2007, 27:827-33.

44. Zervou MI, Sidiropoulos P, Petraki E, et al: Association of a traf1 and a stat4 gene polymorphism with increased risk for rheumatoid arthritis in a genetically homogeneous population. Hum Immunol 2008, 69:567-71.

45. Patsopoulos NA, loannidis JP: Susceptibility variants for rheumatoid arthritis in the traf1-c5 and 6q23 loci: A meta-analysis. Ann Rheum Dis 2009.

46. Kurreeman FA, Rocha D, Houwing-Duistermaat J, et al: Replication of the tumor necrosis factor receptor-associated factor $1 /$ complement component 5 region as a susceptibility locus for rheumatoid arthritis in a european family-based study. Arthritis Rheum 2008, 58:2670-4.

47. Lin PI, Vance JM, Pericak-Vance MA, Martin ER: No gene is an island: The flip-flop phenomenon. Am J Hum Genet 2007, 80:531-8.

48. Graham RR, Cotsapas C, Davies L, et al: Genetic variants near tnfaip3 on $6 \mathrm{q} 23$ are associated with systemic lupus erythematosus. Nat Genet 2008.

49. Kurreeman FA, Goulielmos GN, Alizadeh BZ, et al: The traf1-c5 region on chromosome $9 \mathrm{q} 33$ is associated with multiple autoimmune diseases. Ann Rheum Dis 2009.

50. Behrens EM, Finkel TH, Bradfield JP, et al: Association of the traf1-c5 locus on chromosome 9 with juvenile idiopathic arthritis. Arthritis Rheum 2008, 58:2206-7.

51. Albers HM, Kurreeman FA, Houwing-Duistermaat JJ, et al: The traf1/c5 region is a risk factor for polyarthritis in juvenile idiopathic arthritis. Ann Rheum Dis 2008, 67:1578-80.

52. Hinks A, Eyre $S$, Ke X, et al: Overlap of disease susceptibility loci for rheumatoid arthritis (ra) and juvenile idiopathic arthritis (jia). Ann Rheum Dis 2009.

\section{Pre-publication history}

The pre-publication history for this paper can be accessed here: http://www.biomedcentral.com/1471-2350/12/53/prepub

doi:10.1186/1471-2350-12-53

Cite this article as: Zhu et al:: Single nucleotide polymorphisms at the TRAF1/C5 locus are associated with rheumatoid arthritis in a Han Chinese population. BMC Medical Genetics 2011 12:53.

\section{Submit your next manuscript to BioMed Central and take full advantage of:}

- Convenient online submission

- Thorough peer review

- No space constraints or color figure charges

- Immediate publication on acceptance

- Inclusion in PubMed, CAS, Scopus and Google Scholar

- Research which is freely available for redistribution 